\title{
Experimental studies of neutron-rich nuclei around $N=126$ at KEK isotope separation system
}

\author{
Yutaka Watanabe ${ }^{1, *}$, Yoshikazu Hirayama ${ }^{1}$, Momo Mukai $^{2}$, Murad Ahmed $^{2}$, Yutaka Kakiguchi $^{1}$, Hiroari Miyatake ${ }^{1}$, \\ Michihiro Oyaizu ${ }^{1}$, Peter Schury $^{1}$, Michiharu Wada $^{1}$, Sota Kimura ${ }^{3}$, Hironobu Ishiyama $^{3}$, Sun-Chan Jeong ${ }^{4}$, Jun Young \\ Moon ${ }^{4}$, and Jin Hyung Park ${ }^{4}$ \\ ${ }^{1}$ Wako Nuclear Science Center, Institute of Particle and Nuclear Studies, High-Energy Accelerator Research Organization (KEK), 2-1 \\ Hirosawa, Wako, Saitama 351-0198, Japan \\ ${ }^{2}$ Graduate School of Pure and Applied Science, University of Tsukuba, 1-1-1 Tennoudai, Tsukuba, Ibaraki 305-8571, Japan \\ ${ }^{3}$ Nishina Center for Accelerator-Based Science, RIKEN, 2-1 Hirosawa, Wako, Saitama 351-0198, Japan \\ ${ }^{4}$ Rare Isotope Science Project, Institute for Basic Science (IBS), 70, Yuseong-daero 1689-gil, Yuseong-gu, Daejeon, Republic of Korea
}

\begin{abstract}
Nuclear parameters such as lifetimes and masses of the waiting point nuclei of r-process nucleosynthesis are significant to investigate its astrophysical environment. However, the difficulty in the production of extremely neutron-rich nuclei at the 126 neutron closed shell makes their experimental studies unfeasible. Therefore, the theoretical nuclear models play crucial roles in the simulation of the r-process nucleosynthesis. The systematic nuclear spectroscopy of the neutron-rich nuclei around the 126 neutron closed shell provides significant inputs to those theoretical models to improve their predictability for the waiting point nuclei. We are developing KEK Isotope Separation System (KISS) to perform the systematic nuclear spectroscopy of those neutron-rich nuclei. The nuclei of interest are produced by multi-nucleon transfer reactions between ${ }^{136} \mathrm{Xe}$ and ${ }^{198} \mathrm{Pt}$. The experimental study demonstrated its promising potential to produce them. We have successfully performed the $\beta-\gamma$ spectroscopy and the laser ionization spectroscopy at KISS using the nuclear production by the multi-nucleon transfer reactions.
\end{abstract}

\section{Introduction}

A neutron star merger was discovered by observing gravitational waves in 2017 [1]. The discovery of kilonova coincident with the gravitational waves by observing optical and near-infrared emission made neutron star mergers a promising candidate of the $r$ process site [2]. However the analysis of the light curves and the spectra suggests contributions of several different mass ejection mechanisms from the neutron star merger [3]. To reveal mass and electron fraction of each mass ejecta, the simulation of the r-process nucleosynthesis including properties of relevant $r$ process nuclei is necessary.

Nuclear properties at the 126 neutron closed shell are essential for understanding the r-process in astrophysical nucleosynthesis [4]. The r-process goes through very neutron-rich unstable region, and the nuclei with neutron magic number 126 are considered to be waiting points on the r-process path, which can be progenitors of the peak around the mass number 195 on the observed solar r-abundance distribution. The lifetime and mass of those waiting point nuclei are important parameters to identify the astrophysical environments of r-process. However the area of those nuclei remains unexplored, because it is difficult to access by fusion, fission and fragmentation with stable projectiles and targets.

Thus the theoretical nuclear models play crucial roles in the simulation of the r-process nucleosynthesis. However, predicted $\beta$-decay half-lives of the waiting point nuclei show discrepancies by about one order of magnitude among different theoretical models. Evaluation of isotopic abundance pattern variances from uncertainties of theoretical half-lives indicates significant variations [5]. The reduction of the uncertainties of theoretical predictions of nuclear properties such as halflife and mass is an important issue for comprehensive understanding of r-process nucleosynthesis.

We are now promoting KISS project to produce neutronrich nuclei around neutron number 126 and perform their systematic nuclear spectroscopy such as measurements of lifetime and mass, $\beta-\gamma$ spectroscopy and laser spectroscopy to investigate astrophysical environments of r-process by providing experimental inputs to the theoretical models.

\footnotetext{
Corresponding author: yutaka.watanabe@,kek.jp
} 


\section{Nuclear-production around $N=126$ by MNT reactions}

Recently promising expectations have been focused on multi-nucleon transfer (MNT) reactions to produce neutron-rich nuclei around the 126 neutron closed shell both theoretically and experimentally. Using a macroscopic approach based on Langevin-type dynamical equation of motion, Zagrebaev and Greiner demonstrated that the MNT reaction between the Xe projectile and the $\mathrm{Pb}$ target gave large cross sections enough to produce those neutron-rich nuclei [6]. The experimental measurements of MNT reactions between the $\mathrm{Xe}$ projectile and the $\mathrm{Pb}$ target performed at $\mathrm{ANL}$ indicated a general agreement with the macroscopic calculations [7]. However, experimental data are scarce to evaluate the production of neutron-rich nuclei with neutron number 126, which are located at more neutronrich side of those experimental data for the elements with the atomic number less than 80 .

The GRAZING model [8], which is a semi-classical approach based on the single-particle transfer probabilities, also expects large cross sections of MNT reactions between the ${ }^{136} \mathrm{Xe}$ projectile and the ${ }^{198} \mathrm{Pt}$ target enough to access neutron-rich nuclei around the neutron number 126. We adopted this reaction system to efficiently produce those neutron-rich nuclei via proton pickup and neutron stripping channels. To investigate the feasibility of the reaction system, we performed an experimental study using the large acceptance magnetic spectrometer VAMOS++ [9] coupled to the EXOGAM $\gamma$-ray array [10] at GANIL $[11,12]$. The projectile-like fragments (PLFs) produced in the MNT reactions were detected by the spectrometer and the isotopic distributions of the target-like fragments (TLFs) were deduced from those measurements. The detected $\gamma$-rays confirmed the production of corresponding TLFs. It was observed that the neutron-rich side around neutron number 126 in the isotopic distribution is mainly contributed by the components with total kinetic energy loss (TKEL) less than $75 \mathrm{MeV}$ and the high-TKEL components contribute to the production of neutrondeficient isotopes. Recently, Karpov and Saiko

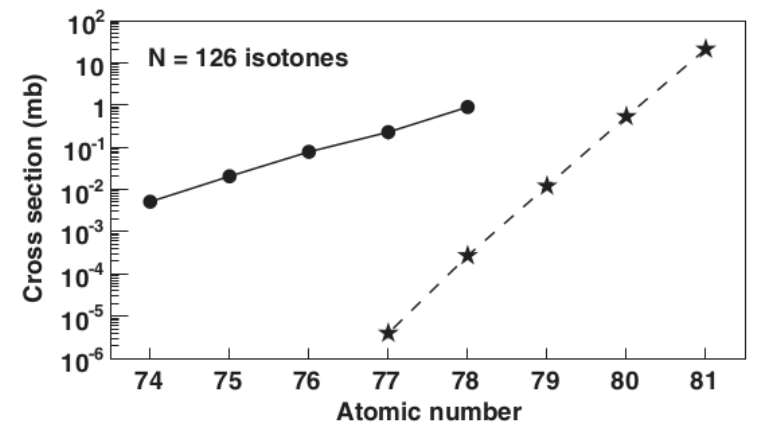

Fig. 1. Cross sections to produce neutron-rich isotones with neutron number 126 by the MNT reactions of ${ }^{136} \mathrm{Xe}$ ( $8 \mathrm{MeV} /$ nucleon) $+{ }^{198} \mathrm{Pt}$ (solid circles) and by the fragmentation reactions between ${ }^{208} \mathrm{~Pb}(1 \mathrm{GeV} /$ nucleon $)$ and Be [14]. (From [12]) demonstrated good agreements between our experimental data and the macroscopic calculations for the total isotopic distributions and decomposed ones in different TKEL values [13]. Figure 1 shows the cross sections to produce neutron-rich isotones with neutron number 126 by the MNT reactions between ${ }^{136} \mathrm{Xe}$ and ${ }^{198} \mathrm{Pt}$ (solid circles) and by the fragmentation reactions between ${ }^{208} \mathrm{~Pb}(1 \mathrm{GeV} /$ nucleon) and $\mathrm{Be}[14]$. Relatively large cross sections as compared with the fragmentation reactions suggests the advantage of the MNT reactions to access neutron-rich nuclei around neutron number 126 [12].

\section{KISS facility}

We have been developing KEK Isotope Separation System (KISS) at RIKEN RIBF facility to separate a single nuclear species among products in the MNT reactions for the nuclear spectroscopy of the neutron-rich nuclei around neutron number 126 [15]. Figure 2 shows a schematic view of KISS. It consists of a gas cell system, a laser system, an extraction chamber, an Isotope Separator On-Line (ISOL) and a detection system for the

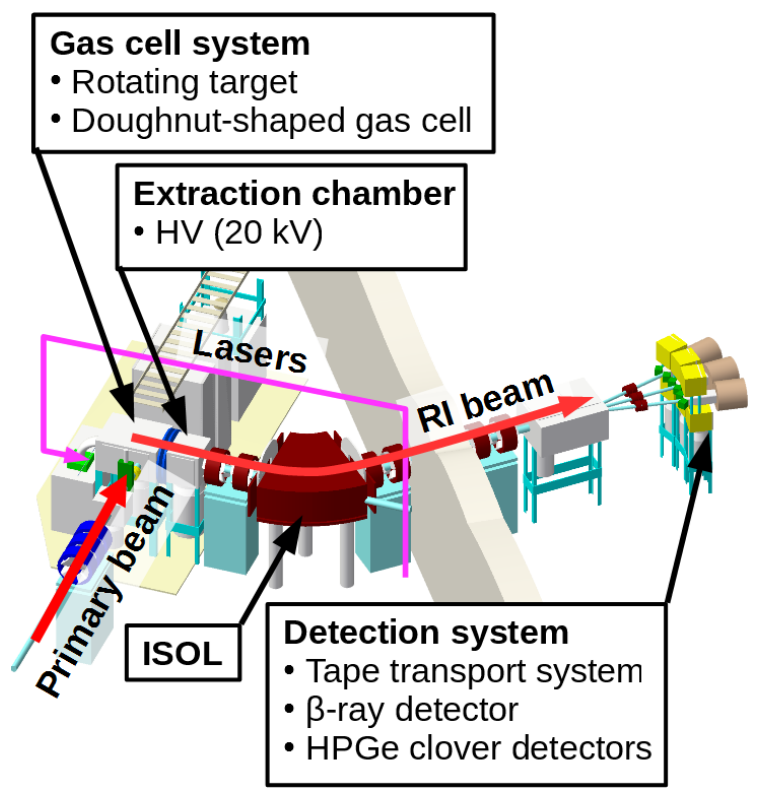

Fig. 2. Schematic view of KEK Isotope Separation System (KISS).

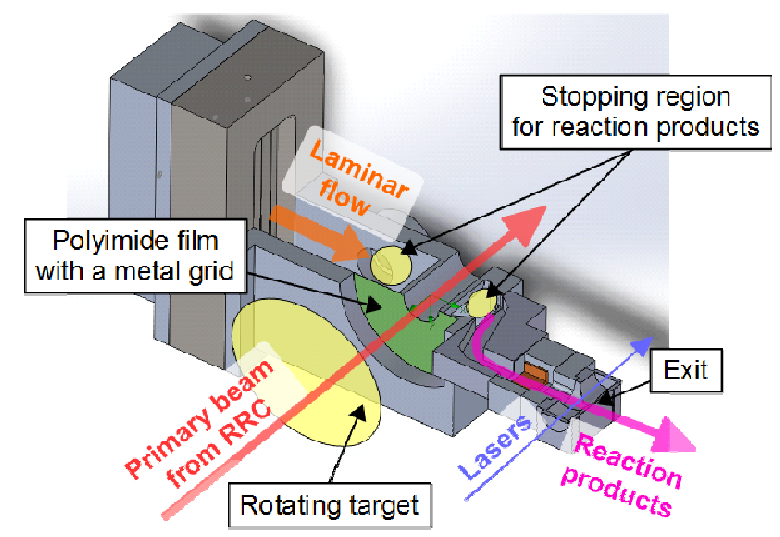

Fig. 3. Doughnut-shaped gas cell. 


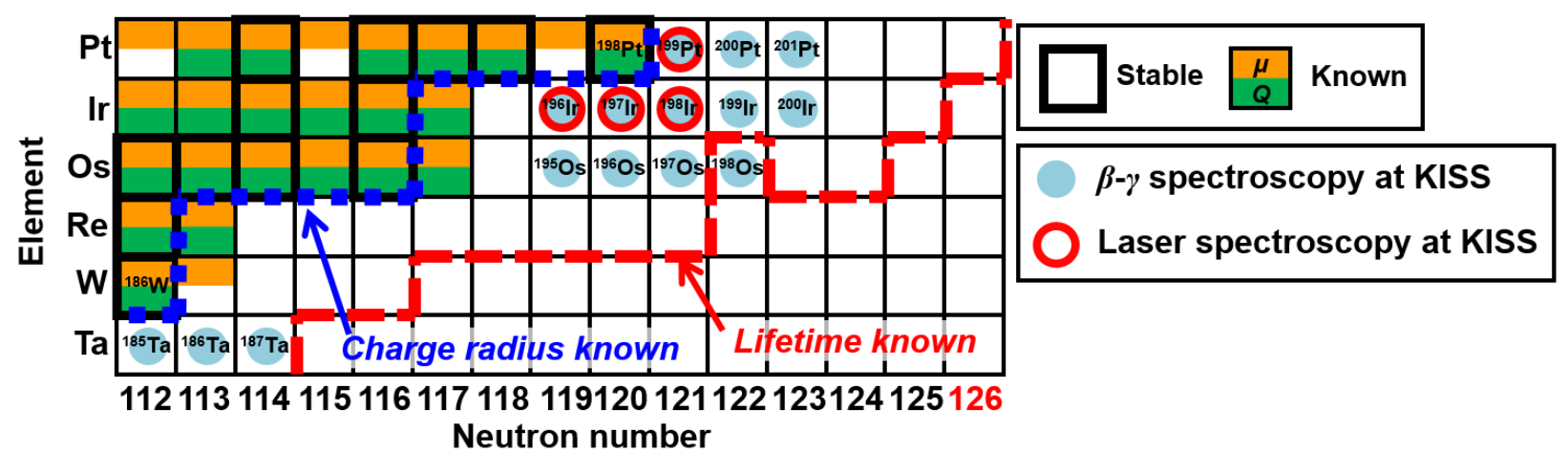

Fig. 4. Radioactive isotopes extracted at KISS. Solid circles and open circles indicate isotopes whose $\beta-\gamma$ spectroscopy and laser ionization spectroscopy was performed, respectively. Isotopes with known magnetic dipole moments $(\mu)$ and electric quadrupole moments $(Q)$ are indicated by boxes filled in upper and lower parts, respectively.

$\beta-\gamma$ spectroscopy. The gas cell system encloses a doughnut-shaped gas cell [16] shown in Fig. 3. The rotating target of ${ }^{198} \mathrm{Pt}$ foils $\left(12.5 \mathrm{mg} / \mathrm{cm}^{2}\right)$ is bombarded by the ${ }^{136} \mathrm{Xe}$ beam $(10.75 \mathrm{MeV} /$ nucleon) from RIKEN Ring Cyclotron after energy-degradation by three layers of titanium foils $(3 \mu \mathrm{m} \times 3)$ to optimize the reaction energies in the target. The TLFs produced by MNT reactions are ejected from the target and go into the gas cell through a window made of a polyimide film $(5 \mu \mathrm{m})$. They are thermalized and neutralized by argon gas filled in the gas cell at the pressure of $88 \mathrm{kPa}$. Those neutral atoms are transported by laminar gas flow, and are element-selectively ionized by two-color two-step laser resonance ionization technique just before the exit of the gas cell. The primary beam passes through the beam pipe with the diameter of $15 \mathrm{~mm}$ through the doughnutshaped gas cell, and is stopped in the water-cooled beam dump. Thus the beam pipe prevents the primary beam from entering the gas cell to suppress the plasma effect induced in the gas by the energy deposit of the primary beam, which would neutralize the laser-ionized atoms of interest resulting in the reduction of their extraction yields. The extracted singly charged ions are transported by multipole RF ion guides and are accelerated by application of a high voltage of $20 \mathrm{kV}$. They are massseparated with the ISOL method, and finally they are transported to the detection system for the $\beta-\gamma$ spectroscopy.

The detection system consists of a tape transport system, a low-background gas counter called Multi-Segmented Proportional Gas Counter (MSPGC) [17] and four HighPurity Germanium (HPGe) clover detectors. The element-selected and mass-separated ions are implanted in the aluminized Mylar tape stretched vertically. The KISS beam is pulsed with certain beam-on and off periods specific for the expected half-lives for $\beta$-decay measurements. The tape is moved in synchronization of the beam pulsing to remove the background from the activity of the accumulated daughter nuclei. The tape is surrounded by the MSPGC to detect $\beta$-rays, which is a two-layered proportional gas counter. Each layer is placed concentrically with the vertical length of $200 \mathrm{~mm}$, and has 16 angularly divided segments. To measure the lifetimes of rare reaction products, high efficiency for the $\beta$-ray detection and the low background rate are essential. The MSPGC achieves $40 \%$ detection efficiency for the $\beta$-rays with $Q_{\beta}$ of $1 \mathrm{MeV}$ including the geometrical acceptable solid angle of $80 \%$ of $4 \pi$. The achieved background rate is as low as $0.1 \mathrm{cps}$. The MSPGC is surrounded by four HPGe clover detectors for the $\gamma$-ray detection with compact geometry of around $5 \mathrm{~cm}$ spacing from the tape. The efficiency for the photo peak detection is $10 \%$ for $\gamma$-rays of $1 \mathrm{MeV}$.

\section{Experimental results}

Figure 4 summarizes experimental results so far at KISS. Solid circles indicate radioactive isotopes which were extracted so far. Twelve kinds of neutron-rich isotopes, ${ }^{199-201} \mathrm{Pt},{ }^{196-200} \mathrm{Ir}$ and ${ }^{195-199} \mathrm{Os}$ were extracted using the ${ }^{198} \mathrm{Pt}$ target, and three kinds of neutron-rich isotopes, ${ }^{185-}$ ${ }^{187} \mathrm{Ta}$, were extracted using a natural $\mathrm{W}$ target [18]. The extracted isotopes were identified by measuring halflives of their $\beta$-decays. The $\beta-\gamma$ spectroscopy of those extracted isotopes was performed.

The dashed lines in Fig. 4 indicate the boundary of known lifetimes before KISS experiments. We have measured the half-life of ${ }^{198} \mathrm{Os}$ for the first time.
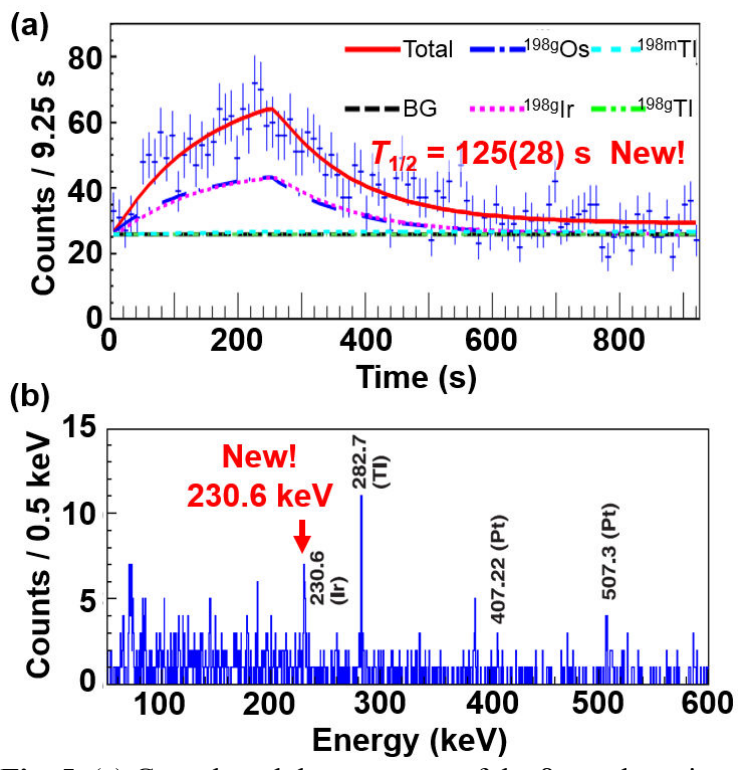

Fig. 5. (a) Growth and decay curves of the $\beta$-ray detection for ${ }^{198} \mathrm{Os}$. (b) $\gamma$-ray energy spectrum coincident with $\beta$ ray detection for ${ }^{198}$ Os. (From [19]) 
Figure 5(a) indicates a time spectrum of detected counts of the MSPGC for the ${ }^{198} \mathrm{Os}$ extraction with the beam-on and off periods of $252 \mathrm{~s}$ and $672 \mathrm{~s}$, respectively. The fitting result with growth and decay curves determined the half-life of the ${ }^{198}$ Os ground state 125(28) s [19]. Figure 5(b) indicates a $\gamma$-ray energy spectrum coincident with $\beta$-ray detection. A $\gamma$-ray peak at the energy of $230.6 \mathrm{keV}$ was newly identified as a $\beta$-delayed $\gamma$ transition of ${ }^{198} \mathrm{Os}$ [19]. The branching ratios and log-ft values for $\beta$-decay branches of ${ }^{198} \mathrm{Os}$ and ${ }^{198} \mathrm{Ir}$ decay chains were evaluated, indicating intense $\beta$-decays among their ground states. Their small log- $f t$ values suggested spin-parity of $0^{-}$or $1^{-}$for the ground state of ${ }^{198} \operatorname{Ir}[19]$.

Figure 6(a) shows the systematics of half-lives of Os isotopes. Solid circles indicate half-lives of ${ }^{196-198}$ Os measured at KISS. Open symbols indicate predictions by different theoretical models and solid squares indicate earlier experimental values. It is obvious that any single theoretical models cannot reproduce all experimental half-life values for osmium isotopes. It would be due to the difficulty of theoretical prediction of half-lives for the neutron-rich nuclei around the 126 neutron closed shell. Figure 6(b) shows the theoretical calculations of single-particle levels of ${ }^{200} \mathrm{~W}[20]$. It indicates the competition between the first-forbidden transitions and the Gamow-Teller transitions. Such a competitive condition makes it difficult to theoretically predict halflives in this nuclear region. More experimental and theoretical studies are required for comprehensive understanding of nuclear structure of those nuclei and for more reliable prediction of nuclear properties for $r$ process nuclei.
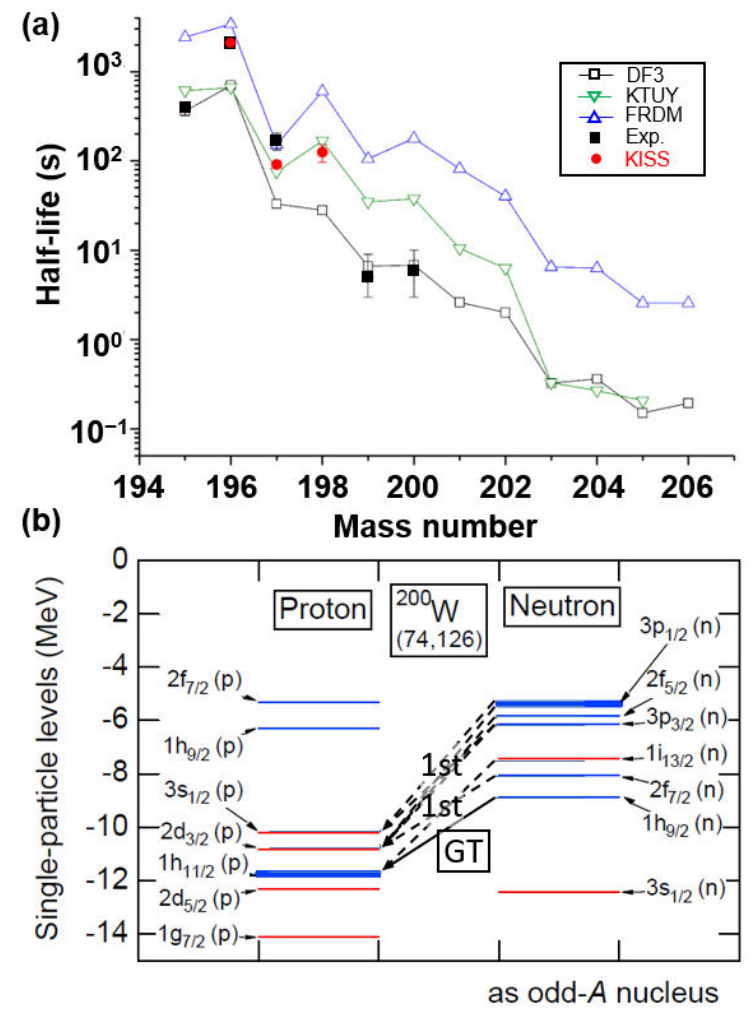

Fig. 6. (a) Systematics of half-lives of Os isotopes. (b) Theoretical calculations of single-particle levels of ${ }^{200} \mathrm{~W}[20]$.
Open circles in Fig. 4 indicate isotopes, ${ }^{199} \mathrm{Pt}$ and ${ }^{196-198} \mathrm{Ir}$, for which the laser ionization spectroscopy was performed at KISS to measure their hyperfine structures (HFS's). HFS measurements provide electro-magnetic moments, which have information about nuclear wavefunctions. Isotope shift, which has information of charge radius, is also obtained, providing hints for the nuclear deformation. The platinum isotopes are discussed to show characteristic shape transition among prolate, triaxial, oblate and finally spherical by approaching to the 126 neutron closed shell [21]. We are performing ingas-cell laser ionization spectroscopy at KISS for study of nuclear structure of neutron-rich nuclei around neutron number 126. Figure 7 shows HFS spectrum by detecting $\beta$-rays for ${ }^{199} \mathrm{Pt}[22]$. The spectrum is contributed by the ground state and the isomeric state of ${ }^{199} \mathrm{Pt}$ since both states are produce in the MNT reactions and extracted at KISS. Using the detected $\gamma$-rays of $392 \mathrm{keV}$, which are associated with the isomeric state decay of ${ }^{199} \mathrm{Pt}$, the spectrum is decomposed to contributions from the ground state ${ }^{199} \mathrm{~g} \mathrm{Pt}$ and the isomeric state ${ }^{199 \mathrm{~m}} \mathrm{Pt}$ of ${ }^{199} \mathrm{Pt}$ by considering the response function of the resonance curve measured for the stable

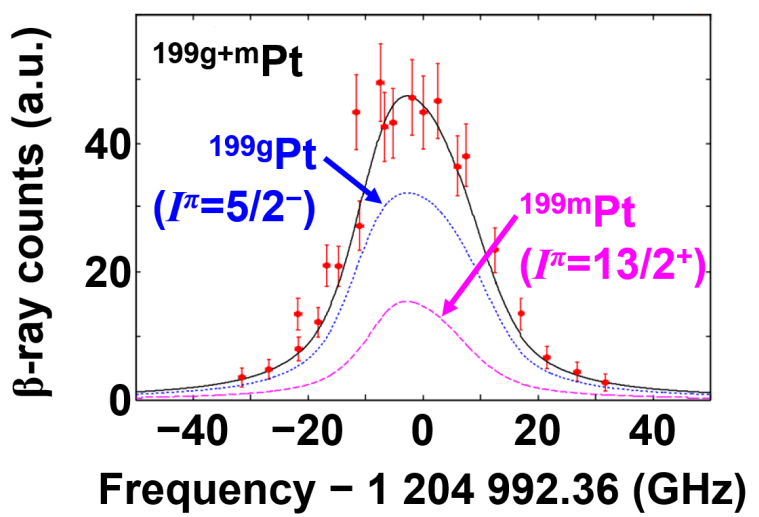

Fig. 7. HFS spectrum by detecting $\beta$-rays for ${ }^{199} \mathrm{Pt}$ [22].

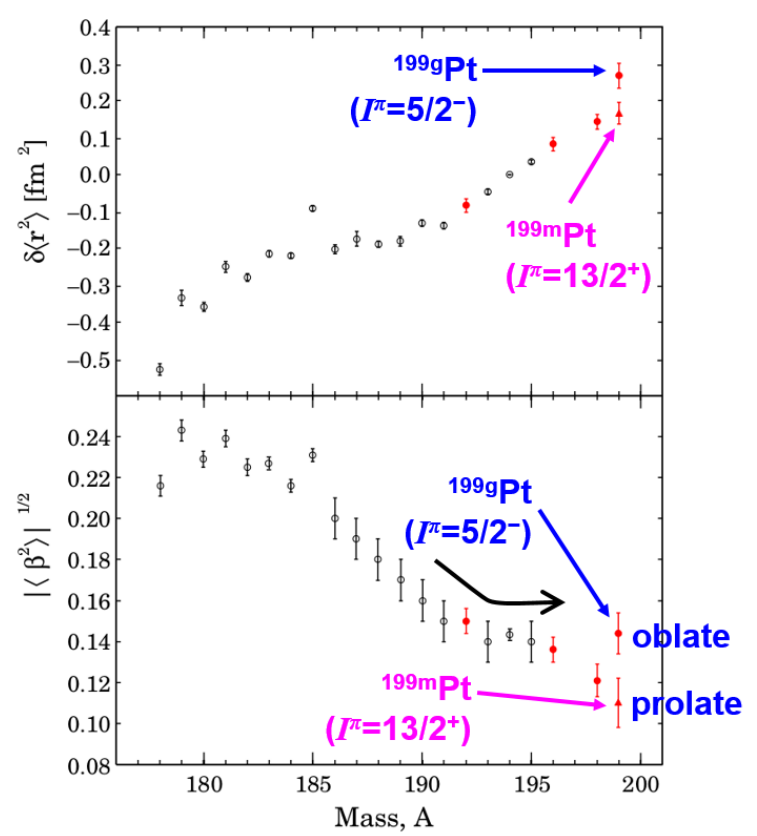

Fig. 8. Systematics of mean square charge radius variation $\delta<r^{2}>$ (upper panel) and quadrupole deformation parameter $\left|<\beta^{2}>\right|^{1 / 2}$ (lower panel) as functions of mass number of $\mathrm{Pt}$ isotopes [22]. 
even-even nucleus ${ }^{198} \mathrm{Pt}$.

Figure 8 shows the systematics of mean square charge radius variation (upper panel) and quadrupole deformation parameter (lower panel) as functions of mass number of $\mathrm{Pt}$ isotopes. The solid circles indicate the ground state values measured at KISS, open circles indicate earlier experimental data. The solid triangle indicate the isomeric state value measured at KISS. The value of the deformation parameter for ${ }^{199} \mathrm{~g} \mathrm{Pt}$ is consistent with the constant value in the systematic trend for $\mathrm{Pt}$ isotopes with mass number lager than 192, indicating oblate deformation. The smaller deformation parameter value for ${ }^{199 \mathrm{~m} P t}$ is consistent with the theoretical prediction, which indicates the prolate deformation [22].

\section{R\&D and future plan of KISS}

For more precise studies of wave-function and deformation, high-precision in-gas-jet laser spectroscopy [23] is required. A new laser system, which consists of a narrow-band laser diode laser, a dye amplifier and intense YAG laser was installed at KISS. A de Laval nozzle was placed at the gas cell exit to form the gas jet. An S-shaped multipole RF ion guide was installed at the downstream of the gas cell to irradiate the excitation laser collinearly along the gas jet with the ionization laser running perpendicularly many times reflected by mirrors in both sides to make the ionization region wide. The off-line test of the in-gas-jet laser ionization spectroscopy using the evaporated atoms from the platinum filament resulted in the Full Width at the Half-Maximum (FWHM) of $0.6(1) \mathrm{GHz}$ in the HFS spectrum for ${ }^{194} \mathrm{Pt}$. The FWHM is obviously reduced from the present value of $12 \mathrm{GHz}$ for in-gas-cell laser ionization spectroscopy. The in-gas-jet laser ionization system is ready for on-line experiments of high-precision laser spectroscopy with refractory elements.

Because the extraction yields become smaller as approaching more and more neutron-rich nuclei in the vicinity of the neutron magic number of 126 , still lower background rate for the $\beta$-ray detection is necessary in order to perform their lifetime measurements. The present MSPGC achieved the background rate of $0.1 \mathrm{cps}$ by enabling two-dimensional tracking on the horizontal plane. We are now adapting the MSPGC to enable threedimensional tracking using resistive $(3 \mathrm{k} \Omega / \mathrm{cm})$ carbon wires for anode wires stretched vertically. The resolution of $2 \mathrm{~mm}$ in FWHM was achieved for the vertical position determination by measuring the charges at both ends of the anode wires. It is good enough to reduce the background rate to the level of $0.01 \mathrm{cps}$.

In addition to the $\beta-\gamma$ spectroscopy, a new branch was constructed in the KISS beam line for the mass measurements. We will use a multi-reflection time-offlight mass spectrograph (MRTOF-MS) for the mass measurements, which achieved the mass resolving power of 150000 in the atomic mass region of 200 [24]. An MRTOF-MS with a helium gas cell ion cooler, a multipole RF ion guide and a mult-trap ion buncher, which accumulate and thermalize the ions extracted from the KISS and bunch them for the MRTOF-MS, were installed already. They are now under commissioning towards the on-line experiment in December, 2019.

\section{Summary}

Systematic nuclear spectroscopy around the 126 neutron closed shell are significant to improve the theoretical predictions of nuclear properties such as lifetimes and masses relevant to r-process nucleosynthesis for identification of its astrophysical environments. The production of those neutron-rich nuclei by MNT reactions has been recently focused on both theoretically and experimentally. However the experimental data had been scarce. We have performed the MNT measurements of ${ }^{136} \mathrm{Xe}$ and ${ }^{198} \mathrm{Pt}$. The results indicated the advantage of the MNT reactions over fragmentation reactions for production of the neutron-rich nuclei around neutron number 126. Encouraged by this results, we have been promoting KISS project, where the MNT reactions of ${ }^{136} \mathrm{Xe}$ and ${ }^{198} \mathrm{Pt}$ are used to produce those neutron-rich nuclei for their systematic nuclear spectroscopy. The gas cell, the laser resonant ionization and the ISOL are used for efficient collection and separation of those MNT reaction products. We have extracted so far fifteen kinds of neutron-rich radioactive isotopes, ${ }^{199-201} \mathrm{Pt},{ }^{196-200} \mathrm{Ir},{ }^{195-208} \mathrm{Os}$ and ${ }^{185-187} \mathrm{Ta}$, and performed lifetime measurements and $\beta-\gamma$ spectroscopy of those nuclei. The laser ionization spectroscopy was performed for ${ }^{199} \mathrm{Pt}$ and ${ }^{196-198} \mathrm{Ir}$. For the precise laser ionization spectroscopy, new in-gas-jet laser ionization system was installed with successful reduction of resonance width in the off-line test. The MSPGC was adapted to enable three-dimensional tracking to reduce the background rate for $\beta$-ray detection down to the level of $0.01 \mathrm{cps}$. It would make possible to perform the $\beta-\gamma$ spectroscopy for neutron-rich nuclei produced in one order of magnitude smaller cross sections than the present setup. The preparation of the MRTOF-MS and the relevant devices for mass measurements of neutronrich nuclei around the 126 neutron closed shell is promoting at KISS. It would be ready soon for the online measurements.

The experiments at the KISS were performed at the RI Beam Factory operated by RIKEN Nishina Center and CNS, University of Tokyo. The authors gratefully acknowledge the staff of accelerators for their support. This work was supported by JSPS KAKENHI Grants No. 232440660, No. 24740180, No. 26247044, No. 15H02096, No. 17 H01132 and No. 18 H03711.

\section{References}

1. B.P. Abbott et al., Phys. Rev. Lett. 119, 161101 (2017)

2. I. Arcavi et al., Nature 551, 64 (2017)

3. M. Tanaka et al., Publ. Astron. Soc. Japan 69, 102 (2017) 
4. E.M. Burbidge et al., Rev. Mod. Phys. 29, 547 (1957)

5. M.R. Mumpower et al., Prog. Part. Nucl. Phys. 86, 86 (2016)

6. V. Zagrebaev and W. Greiner, Phys. Rev. Lett. 101, 122701 (2008)

7. J.S. Barrett et al., Phys. Rev. C 91, 064615 (2015)

8. A. Winther, Nucl. Physics A 572, 191 (1994); 594, 203 (1995)

9. M. Rejmund et al., Nucl. Instrum. and Methods A 646, 184 (2011)

10. J. Simson et al., Acta Phys. Hung. new Ser.: Heavy Ion Phys. 11, 159 (2000)

11. Y.H. Kim et al., EPJ Web of Conferences 66, 03044 (2014)

12. Y.X. Watanabe et al., Phys. Rev. Lett. 115, 172503 (2015)

13. A.V. Karpov and V.V. Saiko, Phys. Rev. C 96, 024618 (2017)

14. T. Kurtukian-Nieto et al., Phys. Rev. C 89, 024616 (2014)

15. Y. Hirayama et al., Nucl. Instrum. and Methods B 353, 4 (2015)

16. Y. Hirayama et al., Nucl. Instrum. and Methods B 412, 11 (2017)

17. M. Mukai et al., Nucl. Instrum. and Methods A 884, 1 (2018)

18. P.M. Walker et al., RIKEN Accel. Prog. Rep. 51, 72 (2018)

19. Y. Hirayama et al., Phys. Rev. C 98, 014321 (2018)

20. H. Koura, JAEA-Review 2010-056, 83 (2010)

21. P. Sarriguren et al., Phys. Rev. C 77, 064322 (2008)

22. Y. Hirayama et al., Phys. Rev. C 96, 014307 (2017)

23. Yu. Kudryavtsev et al., Nucl. Instrum. and Methods B 297, 7 (2013)

24. P. Schury et al., Phys. Rev. C 95, 011305(R) (2017) 